E3S Web of Conferences 2, 04002 (2014)

DOI: $10.1051 / \mathrm{e} 3$ sconf/ 20140204002

(C) Owned by the authors, published by EDP Sciences, 2014

\title{
Human economy and natural economy
}

\author{
Andrea Masullo ${ }^{\mathrm{a}}$ \\ President of the scientific committee of Greenaccord
}

\begin{abstract}
The decline of economy is due to its dependency from a virtual value, the currency, the coin, that in the recent phase of consumerism is so far from real value: human capital and natural capital. If human economy wants to continue to produce wellbeing, it must accept to be a subset of natural economy, intercept flux of matter produced by its circular mechanisms, put constraints in it, i.e. machines and structures, to direct it temporarily for our advantage, and finally release it to the same original flux, in an still usable state. In this way it will assume a function no more parasitic but symbiotic. It will be connected to natural cycles without destroying it, recovering the co-evolutionary link between nature and culture, building an economic web suited to the ecological web; thus we will have a mosaic characterised by biodiversity, technological diversity, and cultural diversity, able to produce a durable prosperity.
\end{abstract}

The economy is a pattern of rules and conventions invented by human being to organise and to drive the trade of goods and values inside the society and among the nations. Ecology is almost the same: it studies the exchange of matter and energy among the species that share a territory. Thus, ecology is the economy of nature, which differ from our economy in having not been invented by man but is embedded into the living and not living matter and determine its functioning.

Universe, according to the big-bang theory, originated about 15 billion years ago. In that instant is generated an enormous quantity of energy that starts to flow toward a progressive degradation, up to reach in the future the maximum level of entropy, the end of its life. It originates from a paradox of science: a situation characterised by quantic numbers null, energy null, volume null, mass null. Science was born $10^{-43}$ seconds after the big-bang, when physical phenomena appearing can be interpreted and codified by laws that after 15 billions of years continue to be valid. Before this moment science is not able to say anything, because it is son and not generator of the big-bang.

Robert Laughlin, Nobel Prize for physics in 1998, says " We live not at the end of discovery but at the end of Reductionism, a time in which the false ideology of human mastery of all things through microscopics is being swept away by events and reason." Laughlin continues saying that he doesn't mean that microscopics are wrong or senseless but only that in many cases become irrelevant in front of the superior laws of organisation that oversee the world. [1]

From the big-bang it starts a process of aggregation of matter and concatenation of phenomena; a propagating organisation process. Starting from big-bang, universe tend to more and more complexity diversifying structures so that diversity of the next possible events increase as fast as it can. The most is matter diversity, the most are the possibility and modality they can aggregate one another and generate further innovations and symmetry breaking. Stuart Kauffman observe in the evolution of

\footnotetext{
${ }^{\text {a }}$ Corresponding author: andrea.masullo@,fastwebnet.it
} 
universe an emerging and ceaseless creativity, the evolutionary emergence of overwhelming organization of process. [2]

The world we can see today is the most recent step of a story of energy degrading and matter selforganising, creating complexity and efficiency; it is a paradoxical story of propagating order that will end in a universal disorder; we are part of this story. The science of complexity, differently by deterministic sciences that describe universe as a big machine driven by physics and chemistry, show us a universe full of autonomous agents self-organizing and self-reproducing. The philosopher Immanuel Kant argued: "An organized being is then not a mere machine, for that has merely moving power, but it possesses in itself formative power of a self-propagating kind, which it communicates to its materials though they have it not of themselves; it organizes them, in fact, and this cannot be explained by the mere mechanical faculty of motion" (Critique of Judgment). The story of universe appears not only as a sequence of happenings determined by natural laws, but a story full of experiences, values, meanings; every agent, we included, seems to be oriented to an emerging, conscious and ceaseless creativity, that continue still now, and only partially can be explained by natural laws.

Everywhere there is a difference that generate a flow of energy or matter, there is always the possibility to build constraints to canalise and transform it in work to modify the environment, creating new opportunities to capture more energy and produce new transformations, building new adjacent possible pathways to be explored. This is doing matter since 15 billion years ago, when elementary particles joined to form nucleus, electrons, and hydrogen atoms, which started to form new atoms and molecules, which joining in organised structures formed organisms capable to capture new flows of energy to transform the environment building new opportunities and new evolutionary pathways: this is the economy of nature.

Our biosphere continue to work linking chemical reactions to produce new structures more and more organised to capture and transform solar energy. Through the photosynthesis it transforms solar radiation in biochemical energy producing glucose, amid and proteins, creating the conditions suitable for developing of living structures independent from photosynthesis, etc. Every new opportunity is taken to open new adjacent scenery for evolution to be explored choosing every time among multiple possible steps. The economy of nature proceed linking cyclic processes, that release matter and energy in the same conditions and at the same point of the cycle from where they have taken it, after the time they remain in a state of "utility"; this allow it to proceed without limits on its road of creation of complexity and efficiency. The natural economy has already achieved very high level of efficiency since we consider that the energy lost by biosphere, about $1,400 \mathrm{GWh} / \mathrm{y}$, is less than one millionth of that it uses. During the 70 million years of Carboniferous, they have been amassed in fossil deposits about 10 billion of GWh, an enormous quantity of energy. Contemporary man is exploiting this opportunity and therefore a citizen of an industrialised country can use more than 200 times the energy needed for his biological sustenance.

Biosphere, in its cyclic proceeding that allow it to continue growing in quality without growing in quantity, don't have limits, as it doesn't exist limits in producing complexity. In figure 1 is showed how passing from ecosystems simple and poor of biodiversity, toward ecosystems complex and reach, they pass from a prevailing capture and dissipation of exergy used for metabolism of the system, to a growth of exergy stored [3]. This in thermodynamic terms reveal the passage from a quantitative development to a qualitative one. Infinite universe different from our could exist

The fact that a qualitative growth don't have limits is easily to demonstrate by a simple calculation as suggested by Kauffman. There are twenty types of amino acids in biology; typical proteins inside our body are made of about three hundred amino acids, even if there are some made by several thousand. So to make a conservative calculation we will consider proteins two hundred amino acids long. Well, the number of different groups of 200 elements sorted from 20 different types is about $10^{260}$ . Suppose it took $10^{-15}$ seconds to make each protein, it would require $10^{67}$ repetitions of the entire history of the universe to create all possible proteins of length two hundred just once. [2]. 
Science and the Future

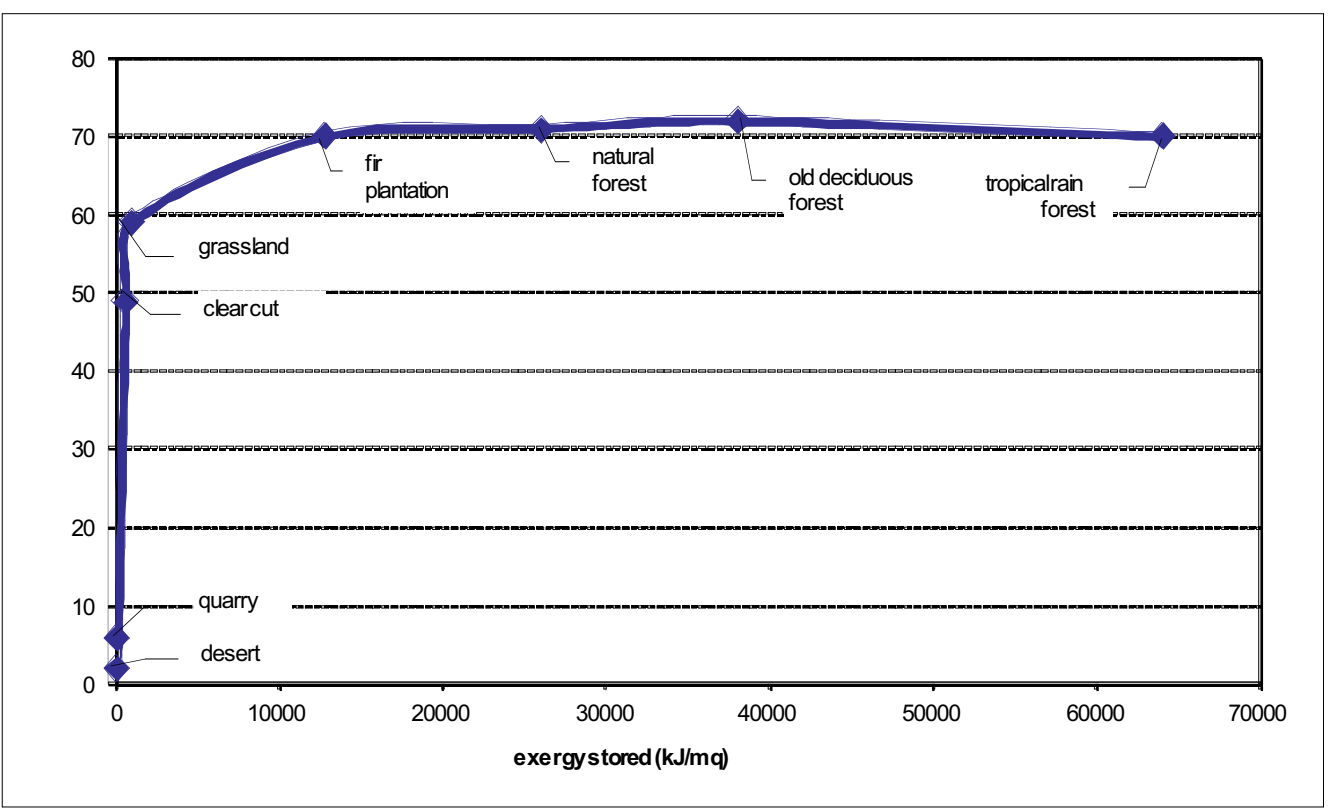

Figure 1. Exergy and evolutionary complexity.

We too, part of this story, produce constraints, canalise flows produced by difference of pressure, temperature, concentration, chemical and electrical potentials, to use their capacity to produce a work, that is to transform the environment. So, water flowing in a river, canalised in a pipe to be delivered to group turbine-generator, is transformed in an electrons flow. The electrons canalised in a light bulb is transformed in a flow of photons, or, if is delivered to supply a computer is transformed in an information flow that can circulate all over the world through internet, remaining in circulation for a time much more longer than that needed by the original water flow to reach the sea losing any potential.

The substantial difference between human and natural economy is that the first one, extracting resources from the cycles created by the second one, and never giving them back, produce damages to natural economy, damages that we call pollution. The linearity of human made processes is the origin of the impassable limits that appear as a fatal destiny in our future. Every process that use resources concentrated and after having used disperse it into the environment, if do not intervene any process or mechanism able to restore the original concentration, will encounter the depletion of the basis of its working principles. Every process, physical or biological, based on a quantitative growth, reach always a saturation level, called climax, and start to oscillate around it indefinitely, until the surrounding conditions don't change. If growth is forced above a critical threshold the system collapse.

In economic and social sphere this has been highlighted in the MIT report "Limits to Growth" [4] on global economy. (figure 2). The depletion of mineral resources easily extractable, already now is determining a strong slowdown of industrial output. The large use of cropland to produce biofuels is already now causing an overexploitation of soils; heavy impact agricultural technics, due to the necessity to feed a growing population, together with a growing use of chemical fertilizer in the attempt to increase a productivity near to saturation, associated with extreme meteorological events more and more conditioned by global climate change, bring us toward the scenario of decrease of food production drawn in figure 2 . The attempt to face the decline of productivity with agricultural practices more invasive will imply a growth of fossil fuel consumption for unit of product. The extraction of fossil fuels from deeper and less achievable deposits, will need more energy for unit of fuel extracted, that is a higher EROEI (energy return on energy invested). This will produce a retrofit that will worsen the situation: higher green-house gas emissions and general pollution. The combination of this factors will produce the decline of world population as indicated by the model, in the first half of this century, or according with other analysis in the second half. This decline will not 
be that wished from the passage to a steady state economy but it will be a consequence of a global decline of the quality of life.

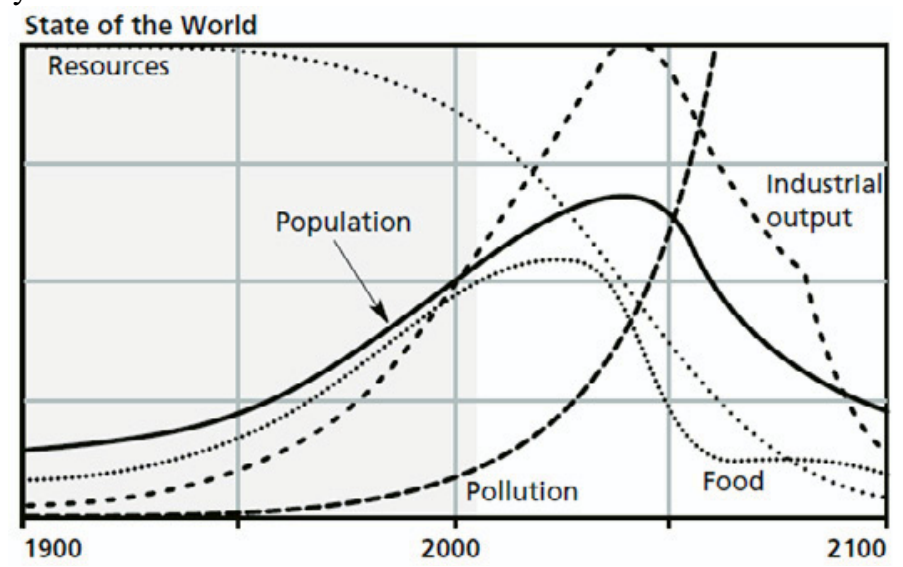

Figure 2. The business as usual scenario from "Limits to growth".

It is a long time we have overtaken the limit of economic growth and entered a regime of antieconomic growth, where marginal costs, that is the negative effects of growth, exceed the benefits. That is what results from the calculation of GPI (genuine progress index), that from ' $80 \mathrm{~s}$ is constant while the negative part of GDP, that related to social and environmental costs, continues to grow [5]. According to the calculation of the global Ecological Footprint, on $20^{\text {th }}$ of August 2013 humanity had already used all the resources that the planet can reproduce all along the year and we emitted all the $\mathrm{CO}_{2}$ it can capture, increasing the ecological debt that we are leaving to the future generations; in other words we use the $35 \%$ more resources and ecosystem services than biosphere can replace, consuming the natural capital existing on our planet.

From what is said above, we realize that if humanity wants to continue to produce wellbeing for future generation and expand it to the whole world population, it must rapidly reform the economic system leaving the unrealistic scenario of unlimited growth of consumption, that have been demonstrated to be damaging and impossible in the long run. The consumption of goods and the industrial production must be limited to goods and products essential or at least able to produce a real wellbeing abolishing wastes and unnecessary goods. The global consumerist model, homogenise trends and consumption, using the same resources and the same technologies; for this reason is extremely vulnerable differently from the economy of nature that funds its success on biodiversity, that makes the ecosystems resilient, i.e. able to adapt to changes, and with high ascendency, i.e. high evolutionary potentiality. Furthermore, the pressing interest of different nations on the same not renewable resources generate inevitably conflicts that will accompany the incoming scarcity of such resources.

But nature overcame five mass extinctions by its extraordinary capacity to produce new organisms and new ecosystems, exactly thanks to its biodiversity, that represents its investment for the future. For almost the whole history of humankind, social relation web have been connected to the ecological web through a co-evolutionary process that produced a great cultural diversity, very precious richness for future progress. The current human economy, uniformly globalised, has a weak adaptive and evolutionary capacity. It is necessary to re-connect economic and ecologic webs through a diversity of technical compatible solutions, so that the social webs restart to improve the human capital, re-valuing the cultural diversity. It is needed a regional mosaic approach to endorse cultural diversity and to drive production and consumptions to a concept of wellbeing specific of each culture and each situation. This will allow us to return in to the evolutionary pattern of the natural economy, where the human economy will be able to develop in quality, producing an unlimited and durable prosperity. 
Science and the Future

\section{References}

1. R. Laughlin, A different universe, reinventing physics from the bottom down, Basic Books, (2005)

2. S.A. Kauffman, Investigations, OUP (2002)

3. A. Masullo, La sfida del bruco, Franco Muzzio editore, (2008)

4. D. Meadows, D. Meadows, J. Randers, Limits to Growth: The 30-Year Update, (2004)

5. I. Kubizewski, R. Costanza et alii, Beyond GDP: Measuring and Achieving Global Genuine Progress (Ecological Economics 93: 57-68) 